\title{
METHODOLOGY FOR STUDYING THE NUMERICAL SPEED OF SOUND IN FINITE DIFFERENCES SCHEMES
}

\author{
Carlos Spa $^{a)}$, Toni Mateos ${ }^{b)}$ and Adan Garriga ${ }^{a), c)}$ \\ a) Department of Information and Communication Technologies \\ University Pompeu Fabra \\ Barcelona, Spain. \\ b)Barcelona Media Innovation Center. Diagonal 177, 08018 \\ Barcelona, Spain. \\ c)Fundació Centre Pitiús d'Estudis Avançats, Palau de Congressos, \\ 07840, Sta. Eulària, Ibiza, Spain. \\ carlos.spa@upf.edu
}

\begin{abstract}
Summary
The space and time discretization inherent to all FDTD schemes introduce non-physical dispersion errors, i.e. deviations of the speed of sound from the theoretical value predicted by the governing Euler differential equations. A general methodology for computing this dispersion error via straightforward numerical simulations of the FDTD schemes is presented. The method is shown to provide remarkable accuracies of the order of $1 / 1000$ in a wide variety of twodimensional finite difference schemes.
\end{abstract}

\section{INTRODUCTION}

Finite-difference time-domain (FDTD) [1] methods provide a simple and accurate way to simulate a wide range of acoustic wave propagation problems: room acoustics analysis, [2, 3] sound scattering from rough surfaces, [4] ultrasounds in biomedical applications, [5] etc.

Over the last decade, much effort has been devoted to improving the accuracy of the FDTD simulations. One important issue common to all FDTD-based algorithms is that they cause a nonphysical dispersion of the simulated waves [6]: due to the discretization of the computational mesh, the actual wave propagation velocity ( $a k a$ numerical speed of sound, or $c_{\text {num }}$ ) is different from the theoretical one, $c$, expected from the governing differential equation of the acoustic field.

A convenient way to parameterize this error is to express the ratio between both velocities in terms of the various parameters relevant to the problem. In this paper we focus on two-dimensional wave propagation in square lattices ( $\Delta x=\Delta y$ ), a case for which it is easy to show that the ratio of velocities, $c / c_{\text {num }}$, can be expressed as a function of only three parameters: the number of cells per wavelenght,
$N_{\lambda}=\lambda / \Delta x$; the angle $\theta$ between the direction of propagation and one of the lattice axis; and the Courant stability number, $S=c \Delta t / \Delta x$.

In some FDTD schemes, the velocities ratio can be well estimated via a simple algebraic manipulation of the FDTD equations, commonly known as Von-Newmann analysis.[1] However, this analytic procedure yields a set of implicit equations that, for non-simple schemes, often become long and cumbersome, making it an impractical path towards illustrating the dependence of $c / c_{\text {num }}$ on the aforementioned parameters. For this reason, it is important to have a means of extracting the velocities ratio from actual simulations, without the need to rely on a priori considerations.

In this paper we present a general numerical methodology for computing $c / c_{\text {num }}$ via simulations of any FDTD algorithm in 2D. In the method presented, a numerical plane wave is employed to validate dispersion relations as originally proposed by An Ping Zhao and Riku M. Mäkinen [7]. In [7] the authors show that a plane wave is more suitable than the commonly used point source $[8,9]$, for the purpose of verifying dispersion relations numerically. Our proposal allow for a statistical determination of the numerical speed of sound and is suitable for any FDTD scheme and for reasonable low values of the number of cells per wavelength.

We apply our methodology to qualitatively different types of FDTD schemes: the original algorithm by K.S. Yee [10] and its recent extensions;[11] the acoustic version of the MacCormack scheme;[12] and the recently developed pseudospectral techniques (PSTD).[13]

The results obtained prove that the methodology provides very accurate computations of $c / c_{\text {num }}$ even for low cells per wavelength. This, together with the fact that it is easily generalizable to $3 \mathrm{D}$, makes it a suitable technique to faithfully research on numerical dispersion errors in the im- 


\section{wavefront}
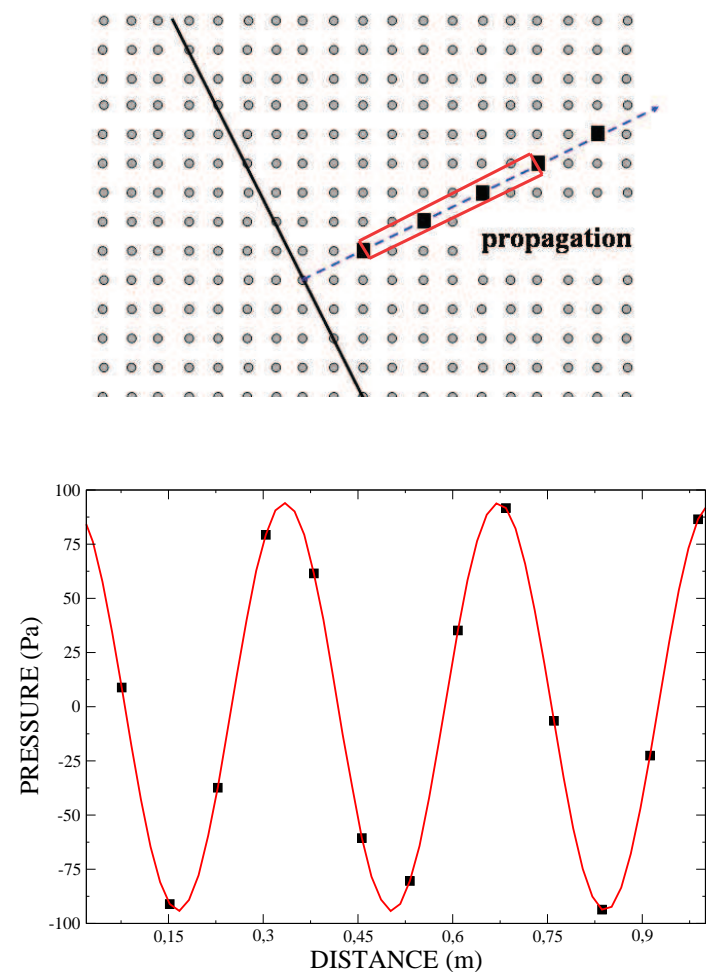

\section{wavefront}
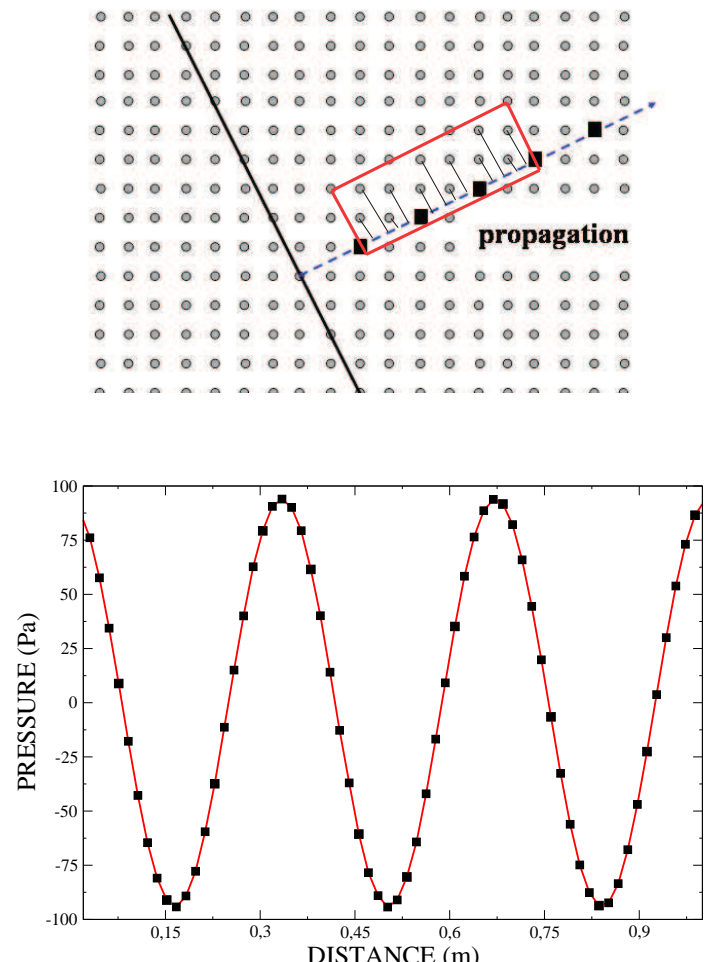

Fig. 1. Upper row: schematic representation of wavefronts propagating within the mesh, at a 26 degrees angle with respect to the axes. Lower row: the result of sampling the pressure field along a line parallel to the propagation direction. Left column: only points directly over such line are considered, leading to a small density of sampling points (square dots). Right column: all other mesh points are projected, leading to a larger sampling density.

plementation of FDTD algorithms in acoustic problems.

The paper is organized as follows: section 2 introduces general methodology; section 3 discusses the results obtained with different FDTD and PSTD methods; finally, section 4 contains the conclusions.

\section{METHODOLOGY}

In this section, we present a general methodology for computing the numerical speed of sound in two-dimensional FDTD schemes. The method consists on creating plane waves traveling in different orientations and following their propagation throughout the mesh. The plane waves are created by exciting a set of mesh points with a time-varying sinusoidal pressure signal $p_{i, j}^{n}=p_{0} \sin (2 \pi \nu n \Delta t)$. Here, $\Delta t$ is the time discretization interval, $n$ is the time step, $(i, j)$ are the two-dimensional spacial coordinates and $\nu$ the frequency of the plane wave. We always set $p_{0}=100 \mathrm{~Pa}$ for illustrative purposes. The orientation of the plane waves, i.e. the angle $\theta$ between the wavefront and the $\mathrm{x}$-axis, is fixed by selecting the excited points to be those for which $\tan \theta=\frac{j}{i}$. This, in turn, implies that the wavefront propagates with velocity parallel to the vector $(j,-i)$.

In order to avoid numerical reflections and to obtain accurate results, we have considered huge lattices ${ }^{1}$, with large Perfectly Matched Layers [14] (PML) at the boundaries. We have chosen PML absorbing boundary conditions not only for their good performance [15] but also for preserving the numerical stability of the algorithms. Finally, we measure the pressure in a region ${ }^{2}$ near the center of the mesh at a time sufficiently short to ensure stationarity. Considering only the pressure at a set of points along the direction of propagation $(j,-i)$ as shown in figure 1 (upper-left hand corner) we obtain a snapshot like the one in figure 1 (lowerleft hand corner). However, it is worth emphasizing that for most angles, the number of points per wavelength obtained by the previous procedure is very small. One simple way to greatly improve the accuracy is to project the pressure

\footnotetext{
${ }^{1}$ Each dimension is about one hundred times the wavelength

${ }^{2}$ The length is about five times the wavelength
} 
C. Spa, T. Mateos and A. Garriga: Dispersion Error in Finite Differences schemes

information of other mesh points to the line $(j,-i)$ perpendicular to the wave front, figure 1 (upper-right hand corner). This leads to a substantial increase on the density of points per wavelength, as observed in figure 1 (lower-right hand corner), and, therefore, to a reduction of the error in $c_{\text {num }}$ associated to the fit.

Finally, by fitting a simple sinusoidal function

$$
p=p_{0} \sin \left(k_{\text {num }} x+\phi\right),
$$

we can easily obtain the numerical wavelength, and therefore the numerical speed of sound,

$$
c_{\mathrm{num}}=\frac{2 \pi \nu}{k_{\mathrm{num}}} .
$$

\section{NUMERICAL VALIDATION}

\subsection{The Yee Algorithm}

The most important basic formulation of the Yee algorithm [10] applied to acoustics was presented in 1994,[2] and further developed for room acoustics in 1995.[3] The Perfectly Matched Layer method for the Yee algorithm was presented also in 1995 [16] for acoustic and elastic modeling.

The scheme is defined on a Cartesian staggered grid with pressure and particle velocity components located at interleaved positions. Spatial and temporal derivatives of the governing partial differential equations are approximated by central-differences leading to second-order accuracy in time and space. In two dimensions, the explicit form is

$$
\begin{aligned}
\left.v_{x}\right|_{i+1 / 2, j} ^{n+1 / 2}= & \left.v_{x}\right|_{i+1 / 2, j} ^{n-1 / 2}-a_{1}\left(\left.p\right|_{i+1, j} ^{n}-\left.p\right|_{i, j} ^{n}\right), \\
\left.v_{y}\right|_{i, j+1 / 2} ^{n+1 / 2}= & \left.v_{y}\right|_{i, j+1 / 2} ^{n-1 / 2}-a_{1}\left(\left.p\right|_{i, j+1} ^{n}-\left.p\right|_{i, j} ^{n}\right), \\
\left.p\right|_{i, j} ^{n+1}= & \left.p\right|_{i, j} ^{n}-a_{2}\left(\left.v_{x}\right|_{i+1 / 2, j} ^{n+1 / 2}-\left.v_{x}\right|_{i-1 / 2, j} ^{n+1 / 2}\right. \\
& \left.+\left.v_{y}\right|_{i, j+1 / 2} ^{n+1 / 2}-\left.v_{y}\right|_{i, j-1 / 2} ^{n+1 / 2}\right),
\end{aligned}
$$

where $a_{1}=\frac{\Delta t}{\rho \Delta x}$ and $a_{2}=\rho c^{2} \frac{\Delta t}{\Delta x}$. A standard VonNewmann analysis[17] yields the following analytic dispersion relation

$$
\begin{aligned}
S^{-2} \sin ^{2}\left(\frac{\pi S}{N_{\lambda}}\right)= & \sin ^{2}\left(\frac{\pi c}{N_{\lambda} c_{\text {num }}} \cos \theta\right) \\
& +\sin ^{2}\left(\frac{\pi c}{N_{\lambda} c_{\text {num }}} \sin \theta\right),
\end{aligned}
$$

which, as explained in the introduction, implicitly relates $c / c_{\text {num }}$ to $S, N_{\lambda}$ and $\theta$. A straightforward analysis of (4) reveals a number of interesting properties: $c / c_{\text {num }}$ tends to 1 in the continuum limit $\left(N_{\lambda} \rightarrow \infty\right)$; the scheme is second order accurate in terms of isotropy;[11] finally, letting $S=$ $\frac{1}{\sqrt{2}}$, which is the maximum value of $S$ for this algorithm to be stable,[2] there is no dispersion error at $\theta=45^{\circ}$.
In order to test our methodology, we compared the values of $c / c_{\text {num }}$ obtained from our simulations to those expected from the analytic equation (4). Figure 2 shows the comparison in two cases with equal $S=1 / \sqrt{2}$, but different $N_{\lambda}$. The continuous lines are obtained from the analytic result (2), whereas dots and error bars were obtained with our methodology after averaging over 15 different different times (snapshots). As it can be seen, the results of the numerical simulations are excellent and the magnitude of error is of order $1 / 1000$ even for $N_{\lambda}=10 \mathrm{cpw}$.
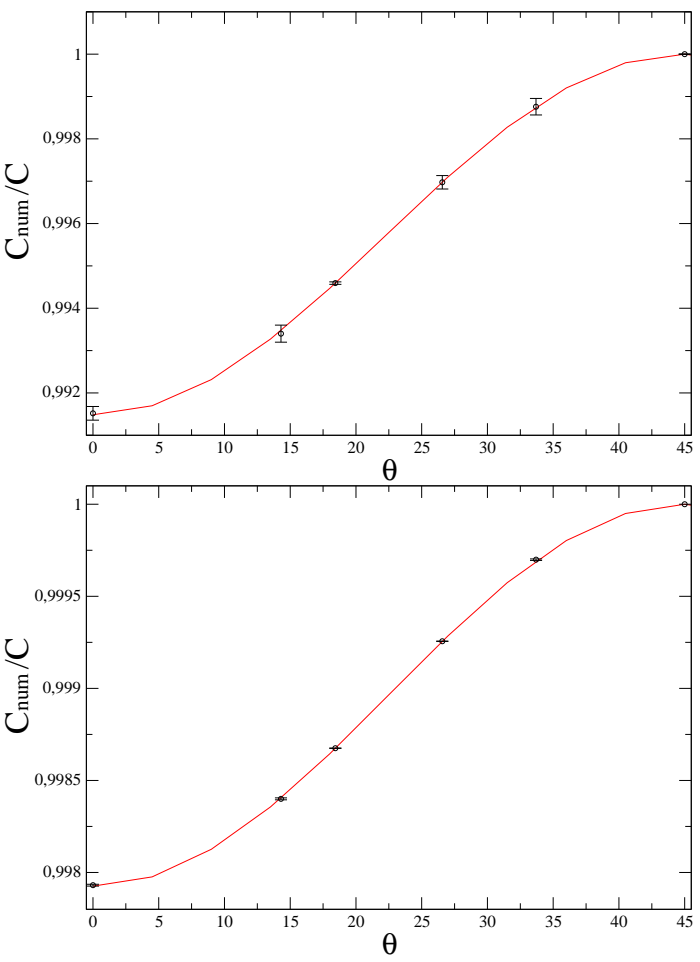

Fig. 2. Analytical (continuous line) and numerical (dots) results for different angles of propagation in Yee's algorithm. Up, $N_{\lambda}=10 \mathrm{cpw}$; down, $N_{\lambda}=20 \mathrm{cpw}$. In both cases $S=1 / \sqrt{2}$.

\subsection{A Family of FDTD algorithms in a staggered grid}

In this section, we discuss a family extensions of Yee's algorithm that improves its accuracy in terms of isotropy.[11] The spatial derivatives employ a combination of differences between nearest neighbors and a next-to-nearest neighbors. 


$$
\begin{aligned}
\left.v_{x}\right|_{i+1 / 2, j} ^{n+1 / 2} & =\left.v_{x}\right|_{i+1 / 2, j} ^{n-1 / 2}-a_{1}\left(\left.p\right|_{i+1, j} ^{n}-\left.p\right|_{i-1, j} ^{n}\right), \\
\left.v_{y}\right|_{i, j+1 / 2} ^{n+1 / 2} & =\left.v_{y}\right|_{i, j+1 / 2} ^{n-1 / 2}-a_{1}\left(\left.p\right|_{i, j+1} ^{n}-\left.p\right|_{i, j-1} ^{n}\right), \\
\left.p\right|_{i, j} ^{n+1} & =\left.p\right|_{i, j} ^{n}-\left(1-\alpha_{2}\right) a_{2}\left(\left.v_{x}\right|_{i+1 / 2, j} ^{n+1 / 2}-\left.v_{x}\right|_{i-1 / 2, j} ^{n+1 / 2}\right. \\
& \left.+\left.v_{y}\right|_{i, j / 2} ^{n+1 / 2}-\left.v_{y}\right|_{i, j-1 / 2} ^{n+1 / 2}\right)-\alpha_{2} a_{2}\left(\left.v_{x}\right|_{i+1 / 2, j+1} ^{n+1 / 2}\right. \\
& +\left.v_{x}\right|_{i+1 / 2, j-1} ^{n+1 / 2}-\left.v_{x}\right|_{i-1 / 2, j+1} ^{n+1 / 2}-\left.v_{x}\right|_{i-1 / 2, j-1} ^{n+1 / 2} \\
& +\left.v_{y}\right|_{i+1, j+1 / 2} ^{n+1 / 2}+\left.v_{y}\right|_{i-1, j+1 / 2} ^{n+1 / 2}-\left.v_{y}\right|_{i+1, j-1 / 2} ^{n+1 / 2} \\
& \left.-\left.v_{y}\right|_{i-1, j-1 / 2} ^{n+1 / 2}\right) .
\end{aligned}
$$

Following Wagner et al. conventions,[11] this family of algorithms is parameterized by a constant, $\alpha_{2}$, within the range $[0,1 / 2]$. It turns out that for the particular value $\alpha_{2}=$ $1 / 6$, the scheme leads to fourth order accuracy in isotropy; more specifically, $c_{\text {num }}=c+\mathcal{O}\left(\Delta x^{6}, \Delta t^{6}\right)$. Moreover, the original scheme by Yee is recovered simply by setting $\alpha_{2}=0$. The dispersion relation following from a VonNewmann analysis reads

$$
\begin{aligned}
& S^{-2} \sin ^{2}\left(\frac{\pi S}{N_{\lambda}}\right)=\left[1-\alpha_{2}+\alpha_{2} \cos \left(\frac{2 \pi c \sin \theta}{N_{\lambda} c_{\mathrm{num}}}\right)\right] \\
& \times \sin ^{2}\left(\frac{\pi c \cos \theta}{N_{\lambda} c_{\mathrm{num}}}\right)+\left[1-\alpha_{2}+\alpha_{2} \cos \left(\frac{2 \pi c \cos \theta}{N_{\lambda} c_{\mathrm{num}}}\right)\right] \\
& \times \sin ^{2}\left(\frac{\pi c \sin \theta}{N_{\lambda} c_{\mathrm{num}}}\right) .
\end{aligned}
$$

We present the values of $c / c_{\text {num }}$ obtained applying our method to this family of algorithms in figure 3. Again, the continuous lines are derived from the analytic result, whereas dots and error bars were obtained with our methodology after averaging over 15 different times. The results obtained have the same accuracy than for the Yee algorithm in all cases tested.

\subsection{The MacCormack Algorithm}

In this section, we apply our methodology to the MacCormack algorithm, which is a two-step explicit method.[17] We have chosen this scheme for different reasons: on the one hand, this is a very popular scheme for dealing with Navier-Stokes equations and its performance for acoustic simulations have been only recently investigated; on the other hand, unlike the algorithms studied in the preceding sections, it uses a centered mesh (i.e. pressure and velocities are computed at the same points of the mesh).

The numerical scheme is obtained integrating the equations of motion in two steps.[12] The first step is given by:
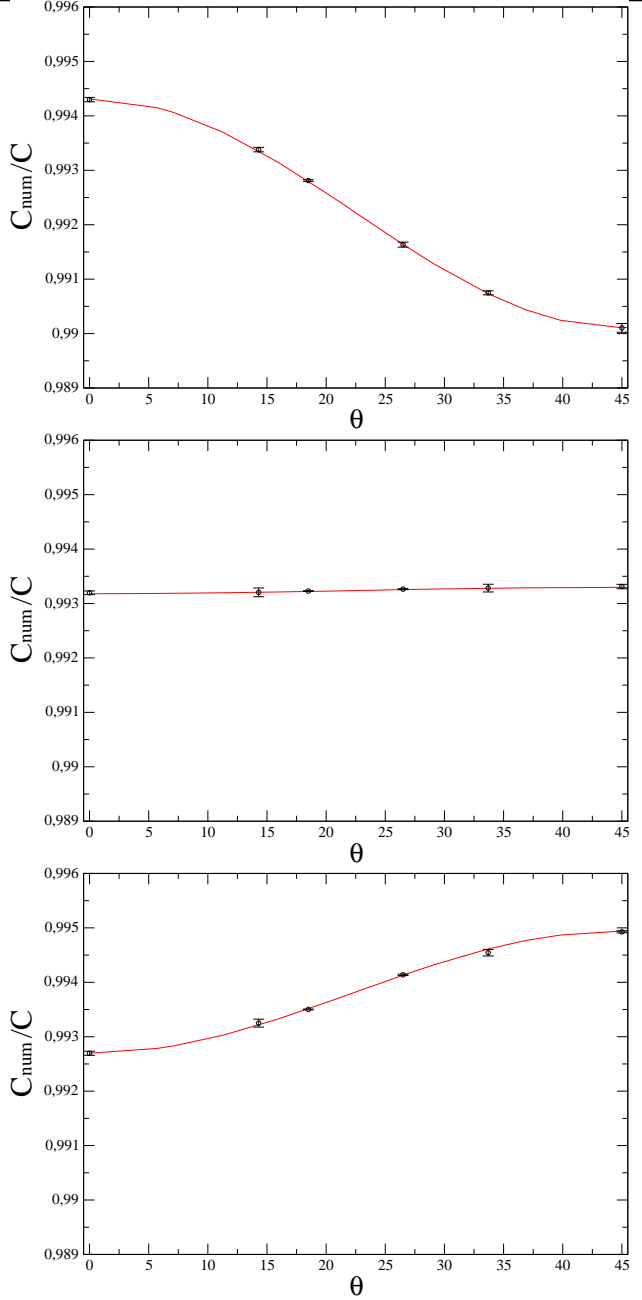

Fig. 3. Analytical (continuous line) and numerical (dots) results for the family of Yee's extensions at the maximum allowed value of $S, N_{\lambda}=10 \mathrm{cpw}$ and $\alpha_{2}=1 / 4$ (up), $\alpha_{2}=1 / 6$ (center) and $\alpha_{2}=1 / 8$ (down).

$$
\begin{aligned}
\left.\widehat{v_{x}}\right|_{i, j} ^{n+1}= & \left.v_{x}\right|_{i, j} ^{n}-a_{1}\left(\left.p\right|_{i+1, j} ^{n}-\left.p\right|_{i, j} ^{n}\right), \\
\left.\widehat{v_{y}}\right|_{i, j} ^{n+1}= & \left.v_{y}\right|_{i, j} ^{n}-a_{1}\left(\left.p\right|_{i, j+1} ^{n}-\left.p\right|_{i, j} ^{n}\right), \\
\left.\widehat{p}\right|_{i, j} ^{n+1}= & \left.p\right|_{i, j} ^{n}-a_{2}\left[\left.v_{x}\right|_{i+1, j} ^{n}-\left.v_{x}\right|_{i, j} ^{n}\right] \\
& -a_{2}\left[\left.v_{y}\right|_{i, j+1} ^{n}-\left.v_{y}\right|_{i, j} ^{n}\right],
\end{aligned}
$$

and the second step 


$$
\begin{aligned}
\left.v_{x}\right|_{i, j} ^{n+1}= & \frac{1}{2}\left[\left.v_{x}\right|_{i, j} ^{n}+\left.\widehat{v_{x}}\right|_{i, j} ^{n+1}\right]-\frac{a_{1}}{2}\left[\left.\widehat{p}\right|_{i, j} ^{n+1}-\left.\widehat{p}\right|_{i-1, j} ^{n+1}\right], \\
\left.v_{y}\right|_{i, j} ^{n+1}= & \frac{1}{2}\left[\left.v_{y}\right|_{i, j} ^{n}+\left.\widehat{v_{y}}\right|_{i, j} ^{n+1}\right]-\frac{a_{1}}{2}\left[\left.\widehat{p}\right|_{i, j} ^{n+1}-\left.\widehat{p}\right|_{i, j-1} ^{n+1}\right], \\
\left.p\right|_{i, j} ^{n+1}= & \frac{1}{2}\left[\left.p\right|_{i, j} ^{n}+\left.\widehat{p}\right|_{i, j} ^{n+1}\right]-\frac{a_{2}}{2}\left[\left.\widehat{v_{x}}\right|_{i, j} ^{n+1}-\left.\widehat{v_{x}}\right|_{i-1, j} ^{n+1}\right] \\
& -\frac{a_{2}}{2}\left[\left.\widehat{v_{y}}\right|_{i, j} ^{n+1}-\left.\widehat{v_{y}}\right|_{i, j-1} ^{n+1}\right] .
\end{aligned}
$$

Finally, its analytic dispersion relation is complex enough to render it almost unusable. In cases like this, our methodology presents a simpler and more straightforward way to study the dispersion error. For the sake of clarity of exposition, we have left the explicit equations of MacCormack's dispersion relation for the Appendix.

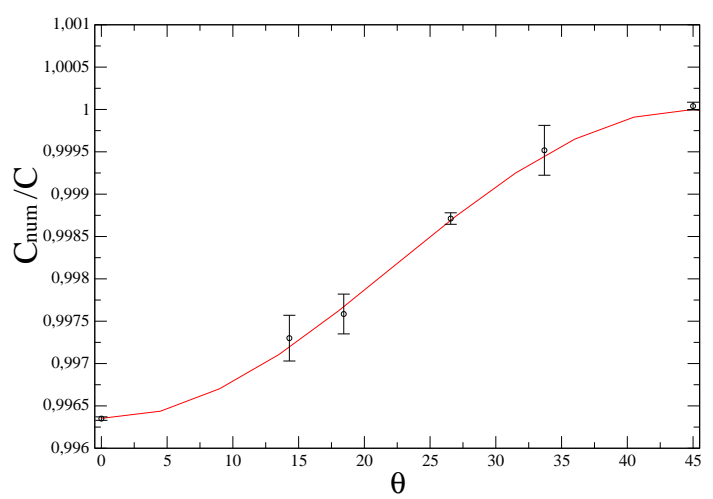

Fig. 4. Comparison of analytical (continuous line) and numerical (dots) results for MacCormack's algorithm, with $N_{\lambda}=30 \mathrm{cpw}$ and $S=1 / \sqrt{2}$.

The comparison with our methodology is presented in figure 4, where excellent agreement is observed again. As can be shown in figure 4 we have obtained again accuracies of the order of 1/1000 averaging only over 15 snapshots.

\subsection{PSTD Method}

The FDTD methods are a simple, robust and powerful technique to simulate transient acoustic phenomena. However, the standard lore indicates that a spatial sampling density above $10-15$ cells per wave-length is needed to produce accurate results. This problem becomes even more critical in large acoustic problems (such as rooms with long reverberation times), making it necessary to increase the spatial sampling rate beyond this range to reduce the cumulative numerical dispersion error.[1]

PSTD methods improve this situation significantly by using more refined approximations for spatial derivatives. For example, those PSTD methods based on Fourier transforms essentially use all the points in a given row of the mesh to approximate the partial derivative along the row direction. In two dimensions, the explicit form of the PSTD scheme is

$$
\begin{aligned}
\left.v_{x}\right|_{i, j} ^{n+1 / 2}= & \left.v_{x}\right|_{i, j} ^{n-1 / 2}-\frac{\Delta t}{\rho} \mathcal{F}_{x}^{-1}\left(\iota \frac{2 \pi n_{x}}{N_{x} \Delta x} \mathcal{F}_{x}\left(\left.p\right|_{:, j} ^{n}\right)\right), \\
\left.v_{y}\right|_{i, j} ^{n+1 / 2}= & \left.v_{y}\right|_{i, j} ^{n-1 / 2}-\frac{\Delta t}{\rho} \mathcal{F}_{y}^{-1}\left(\iota \frac{2 \pi n_{y}}{N_{y} \Delta y} \mathcal{F}_{y}\left(\left.p\right|_{i,:} ^{n}\right)\right), \\
\left.p\right|_{i, j} ^{n+1}= & \left.p\right|_{i, j} ^{n}-\rho c^{2} \Delta t \mathcal{F}_{x}^{-1}\left(\iota \frac{2 \pi n_{x}}{N_{x} \Delta x} \mathcal{F}_{x}\left(\left.v_{x}\right|_{:, j} ^{n+1 / 2}\right)\right) \\
& -\rho c^{2} \Delta t \mathcal{F}_{y}^{-1}\left(\iota \frac{2 \pi n_{y}}{N_{y} \Delta y} \mathcal{F}_{y}\left(\left.v_{y}\right|_{i,:} ^{n+1 / 2}\right)\right),(9)
\end{aligned}
$$

where $\mathcal{F}_{\mu}$ and $\mathcal{F}_{\mu}^{-1}$ denote the Fourier transform over the $\mu$-axis and its inverse respectively; $n_{x}$ and $n_{y}$ are the index of the Fourier transforms, and the : symbol denotes all $\mu$ coordinate along the straightline cut through the space lattice. Finally, $\iota=\sqrt{-1}$ and $N_{\iota}$ are the total grid points over the $\mu$
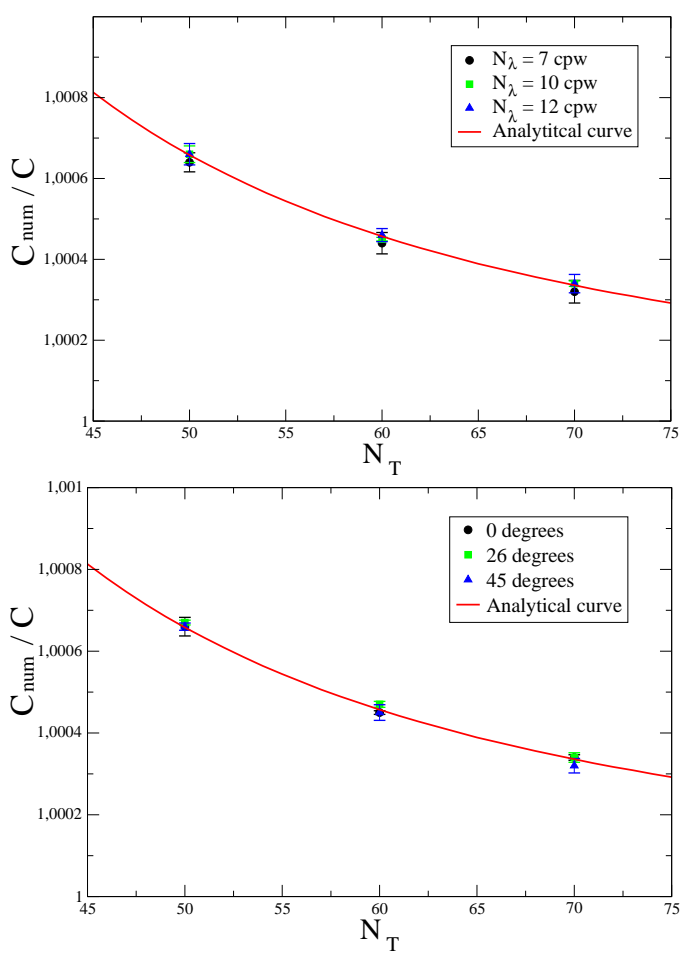

Fig. 5. Analytical curve (continuous line) compared to numerical simulations (dots). Up: dispersion error versus time discretization $N_{T}$ for different $N_{\lambda}$, at a fixed angle $\theta=0$. Note the remarkable independence of the results of $N_{\lambda}$. Down: dispersion error versus $N_{T}$ for different angles, at fixed $N_{\lambda}=10 \mathrm{cpw}$.

A direct consequence of the expression (9) is that the 
C. Spa, T. Mateos and A. Garriga: Dispersion Error in Finite Differences schemes

stability criterium does not depend on $N_{\lambda}$, which may be set to a value as low as 2. Similarly, from a dispersion error point of view, PSTD methods exhibit a perfectly isotropic relation, i.e. the ratio $c / c_{\text {num }}$ does not depend on the propagation angle $\theta$. More specifically, for large enough numerical domains:

$$
\frac{c_{\mathrm{num}}}{c}=\frac{\pi}{N_{T} \sin \left(\frac{\pi}{N_{T}}\right)}, \quad N_{T}:=\frac{T}{\Delta t},
$$

where $N_{T}$ controls the time discretization, $T$ being the period of the planar wave. Note that, just like the stability criterium, the dispersion error does not depend on the spatial discretization $N_{\lambda}$ either.

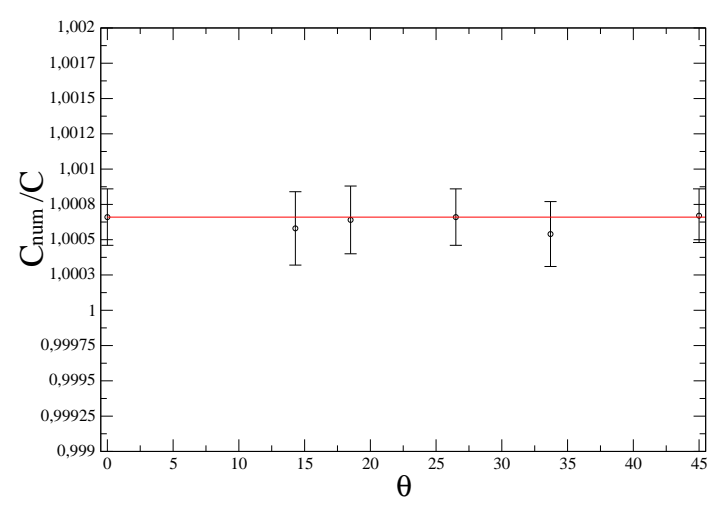

Fig. 6. Dispersion error versus angle at fixed $N_{\lambda}=10 \mathrm{cpw}$, $N_{T}=50$. Note the almost perfect isotropy.

The results of our methodology applied to the Fourier transform PSTD scheme are compared to the analytical ones derived from (10) are shown in figures 5 and 6 . Once more, the agreement proves to be excellent in all the situations studied.

\section{CONCLUSIONS}

In this letter we have presented a general statistical methodology for studying and validating the dispersion error of generic FDTD algorithms by direct computation of the numerical speed of sound. The procedure is simple but powerful, as it easily generates enough data to obtain computationally accuracies of the order of $1 / 1000$. We have shown that it applies equally well to a wide variety of qualitatively different schemes in 2D: FDTD in staggered and non-staggered meshes, isotropic extensions of FDTD, and pseudospectral techniques in time domain. We also have shown that the methodology works excellently even at low values of the number of cells per wavelength.

Finally, the methodology extends rather straightforwardly to three-dimensional cubic meshes. To create plane waves, all that is needed is to excite a whole plane of the 3D mesh. The normal to the plane corresponds to the propagation direction, which can be used to sample the pressure field as described in section 2 , fit a sinusoidal function, and easily obtain the behavior of $c / c_{\text {num. }}$.

\section{ACKNOWLEDGMENTS}

The authors wish to thank Vicente López, Jaume Durany, Pau Arumí, David García and Jordi Arqués for useful discussions. C. S. has been suported by a grant of the University of Pompeu Fabra. T. M. acknowledges funding from the 'Programa Torres y Quevedo' of the Spanish Ministerio de Educación y Ciencia.

\section{REFERENCES}

[1] A. Taflove and S. Hagness. Computational Electrodynamics: The finite-difference time-domain method. Artech House, 2005.

[2] D. Botteldoren. Acoustical finite-difference timedomain simulation in a quasi-Cartesian grid. Acoustical Society of America, 95: 2313-2319, 1994.

[3] D. Botteldoren. Finite-difference time-domain simulations of low-frequency room acoustic problems. Acoustical Society of America, 98:3302-3308, 1995.

[4] J. B. Schneider, F. D. Hastings and S. L. Broschat. A finite-difference time-domain solution to scattering from a rough pressure-release surface. The Journal of the Acoustical Society of America, 102:3394-3400, 1997.

[5] I. M. Hallaj and R. O. Cleveland . FDTD simulation of finite-amplitude pressure and temperature fields for biomedical ultrasound. The Journal of the Acoustical Society of America, 105:L7-L12, 1999.

[6] L. N. Trefethen. Group velocity in finite difference schemes. SIAM Review, 24:113-136, 1982.

[7] A. P. Zhao and R. M. Mäkinen. An accurate and logically correct way to verify the numerical dispersion relations of FDTD and ADI-FDTD methods. Microwave and Optical Technology Letters, 40(5):427-431, 2004.

[8] T. Namiki and K. Ito. Investigation of numerical errors of the two-dimensional ADI-FDTD method. IEEE Trans. Microwave Theory Techniques, 48:1950-1956, 2000.

[9] G. Sun and C. W. Trueman. Analysis and numerical experiments on the numerical dispersion of twodimensional ADI-FDTD method. IEEE Antennas and Wireless Propagation Letters, 2:78-81, 2003. 
[10] K. S. Yee. Numerical solution of initial boundary values Problems involving Maxwell equations in isotropic media. IEE Transactions on antennas and propagation, 14:302-307, 1966.

[11] C. Wagner and J. Schneider. An acoustic finitedifference time-domain algorithm with isotropic dispersion. Journal of Computational Acoustics, 13(2):365384, 2005.

[12] A. Garriga, C. Spa and V. López. Computation of the complete acoustic field with finite-difference algorithm. Proceeding of the Forum Acusticum 2005. Budapest.

[13] Q. H. Liu. The PSTD algortihms: a time-domain method requiring only two cells per wavelenght. $\mathrm{Mi}$ crowave and Optical Technology Letters, 15:158-165, 1997.

[14] J-P Berenger. Three-Dimensional Perfectly Matched Layer for the Absorption of electromagnetic waves. Journal of Computational Physics.127,363-379, 1996.

[15] X. Yuan, D. Borup, J.W. Wiskin, M. Berggren, R. Eidens and S.A. Johnson. Formulation and Validation of Berenger's PML absorbing boundary for the FDTD simulation of acoustic scattering. IEEE Trans. on Ultrasonics, Ferroelectrics and Frequency Control, 44:816822, 1997.

[16] J. G. Maloney and K. E. Cummings. Adaptation of FDTD techniques to acoustic modeling. Proceedings of the Applied Computational Electromagnetics at the Naval Postgradurate School. 724-731, 1995.

[17] J.D. Hoffman. Numerical Methods for Engineers and Scientists. MacGraw-Hill, 1992.

\section{A. DISPERSION RELATION OF THE MACCORMACK ALGORITHM}

In section 3.3 we applied our methodology to the MacCormack algorithm: a two-step explicit method.[17] A standard Von-Newmann analysis leads to the following dispersion relation:

$$
\begin{aligned}
& 0=-\frac{1}{4} e^{-\iota k \Delta(\cos \theta+\sin \theta)}\left(-1+e^{\iota k \Delta x \cos \theta}\right)^{2}(-1 \\
& \left.+e^{\iota k \Delta x \sin \theta}\right)^{2} S^{4}+\left(1-e^{-\iota \omega \Delta t} S^{2}[-1\right. \\
& +\cos (k \Delta x \cos \theta)])\left(1-e^{-\iota \omega \Delta t}+S^{2}[-1\right. \\
& +\cos (k \Delta x \sin \theta)])\left(1-e^{-\iota \omega \Delta t} S^{2}[-2 \cos (k \Delta x \cos \theta)\right. \\
& +\cos (k \Delta x \sin \theta)])+S^{2} \sin ^{2}(k \Delta x \cos \theta)[[1 \\
& \left.-e^{-\iota \omega \Delta t} S^{2}(-1+\cos (k \Delta x \sin \theta))\right] \sin (k \Delta x \cos \theta) \\
& -\frac{1}{2} e^{\iota k \Delta x \cos \theta}\left(-1+e^{\iota k \Delta x \cos \theta}\right)\left(-1+e^{\iota k \Delta x \sin \theta}\right) \\
& \left.\times \quad S^{2} \sin (k \Delta x \sin \theta)\right]-\frac{1}{2} S^{2} \sin (k \Delta x \sin \theta)\left(S^{2}\right. \\
& \times \sin (k \Delta x \cos \theta)\left[-1+e^{\iota k \Delta x \cos \theta}\right]\left[-1+e^{\iota k \Delta x \sin \theta}\right] \\
& -2\left[-1+e^{-\iota \omega \Delta t}+S^{2}-S^{2} \cos (k \Delta x \cos \theta)\right] \\
& \times \quad \sin (k \Delta x \sin \theta)),
\end{aligned}
$$

where $\iota=\sqrt{-1}$ and $k$ is the numerical wavenumber of the plane waves. The equality of (11) can only be satisfied for complex values of $k=\alpha+\iota \beta$. The velocity of propagation of the simulated wave is then related to the real part of $k$ via $c_{\text {num }}=\frac{2 \pi \nu}{\alpha}$. On the other hand, the imaginary part of $k$ corresponds to a (mild) exponential damping of the plane waves along their propagation in the mesh. Its nonzero value is due to the fact that the MacCormack scheme is not perfectly conservative. Note that (11) is a rather complicated implicit equation for $c_{\text {num }}$. 